\title{
New Approaches to Indoles and Indole Alkaloids
}

\author{
Peter Wipf* and Filip Petronijević \\ Department of Chemistry, University of Pittsburgh, Pittsburgh PA 15260 \\ *pwipf@pitt.edu
}

Keywords: indoles, Diels-Alder cycloaddition, alkaloids

\section{INTRODUCTION}

Second only to pyridines, indoles are among the most common aromatic scaffolds present in bioactive molecules. The 5-hydroxy indole moiety alone has currently $>10,500$ substructure hits Among the many indole-containing alkaloid scaffolds, ergot alkaloids comprise a notable group of natural products of great biological activities. The striking biological properties of indoles and challenging polycyclic molecular architectures and wide spectrum of physiological activities of indole derivatives have attracted organic chemists for decades. In spite of all the synthetic approaches published to date, there is still opportunity and need for novel synthetic strategies that can harness the unique chemical diversity of indole derivatives.

\section{RESULTS AND DISCUSSION}

We have previously shown that 4-mono- and 3,4disubstituted indoles can be synthesized through an intramolecular Diels-Alder cycloaddition of furan (IMDAF) reaction. Recently, we have accomplished an extension of this procedure to the direct preparation of 5-hydroxy indoles. In addition, we have been able to demonstrate the utility of our indole methodology for the construction of the indole natural product cycloclavine. We were attracted to this compound as a synthetic target due to its unusual molecular scaffold, featuring the only cyclopropane-containing ergot alkaloid. Our $1^{\text {st }}$ generation retrosynthetic plan for cycloclavine assumed that the stability of the cyclopropane moiety in the hydroindole intermediate $\mathbf{2}$ was sufficient to allow a thermal [4+2] process, and that dienone 3 could be obtained by a cascade TBS-deprotection-intramolecular $\mathrm{S}_{\mathrm{N}} 2$-displacement (Figure 1). Indolinone 4 was be formed by ortho-alkylation of 3-aminophenol $\mathbf{5}$. For the selective hydrogenation of cross-conjugated dienone $\mathbf{3}$, we hoped that we could effect this conversion by taking advantage of Lewisacidic reducing agents and the electron-donating properties of the $\beta$-amino substituent. While we were able to complete this $1^{\text {st }}$ generation approach, it resulted in the preparation of a diastereomer-5-epicycloclavine-of the synthetic target. We were able to revise this analysis successfully, according to the pathway shown in Figure 2.
Figure 1. $1^{\text {st }}$ Generation Analysis of Cycloclavine.

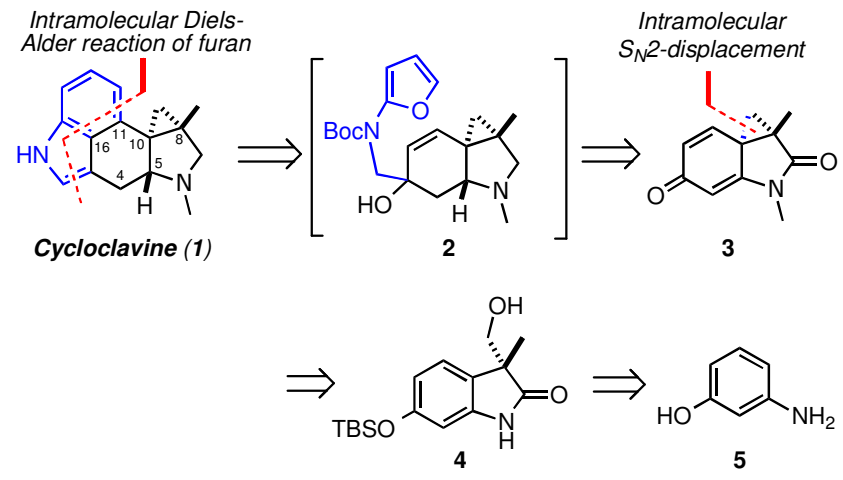

Figure 2. $2^{\text {nd }}$ Generation Retrosynthetic Analysis.

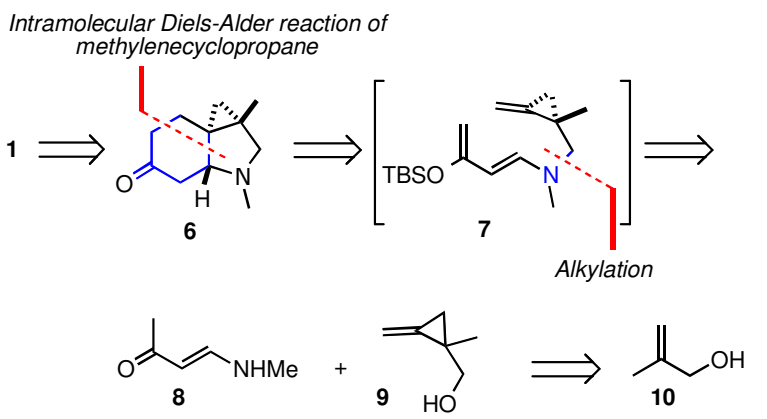

\section{CONCLUSION}

As an extension of our new indole methodologies, we developed a novel synthetic route to the ergot alkaloid cycloclavine, proceeding in 14 steps and $1.2 \%$ overall yield. Noteworthy features of this work include the formation of the indole moieties through the allylic alcohol-IMDAF reaction, as well as the rapid synthesis of cycloclavine's indoline core through a novel and highly stereoselective intramolecular Diels-Alder reaction of a methylenecyclopropane.

\section{ACKNOWLEDGEMENTS}

This work was supported by the NIH/NIGMS CMLD program (GM067082).

\section{REFERENCES}

Petronijević, F.; Timmons, C.; Cuzzupe, A.; Wipf, P. Chem. Commun. 2009, 1, 104.

${ }^{2}$ Petronijević, F.; Wipf, P. J. Am. Chem. Soc. 2011, 133, in press. 